\title{
Liquefied Petroleum Gas (LPG) Leakage Detection and Monitoring System
}

\author{
Tamil Selvi a/p Sitan ${ }^{1}$ and Aimi Syamimi Ab Ghafar ${ }^{1 *}$ \\ ${ }^{1}$ Department of Electrical Engineering Technology, Faculty of Engineering Technology, \\ University Tun Hussein Onn Malaysia, Pagoh, Johor, Malaysia
}

Received 29 April 2018; accepted 21 November 2018; available online 19 December 2018

DOI: https://10.30880/jst.2018.10.03.007

\begin{abstract}
A system consists of detection and monitoring system is very common in industrial field. However, this system still very rare in domestic. Detection and monitoring system of Liquefied Petroleum Gas (LPG) is very important in home because it is common to be used at homes. The purpose of this project is to develop Liquefied Petroleum Gas leakage detection and monitoring system. This system consists of two sensing unit, two types of alarm alert and an online monitoring system. Gas sensor is used for gas leakage detection specified to LPG gas only and load cell sensor is used to weigh the gas cylinder. A physical alarm alert using buzzer is installed for gas detection only while non-physical alert alarm which is sending notification and email to user through smartphone are developed to equip the system with Internet of Thing (IoT) technology. This includes notification of both gas leakage and empty gas cylinder. For monitoring purpose, Blynk application is used as the IoT platform because of its user friendliness for both iOS and Android smartphone users. Real-time data is sent through WiFi so that users can monitor their kitchen condition remotely and receive alert for both gas leakage and gas cylinder condition through their smartphones. In conclusion, the proposed LPG leakage detection and monitoring system has been successfully developed and has lower cost compared to existing system on the shelves. This system is suitable for domestic usage and small premise usage.
\end{abstract}

Keyword: LPG leakage detection; monitoring gas cylinder; IoT; alert.

\section{Introduction}

Nowadays, the usage of Liquefied Petroleum Gas (LPG) in Malaysia is widely used in many fields such as in homes, restaurants, industries (small scale to big scale) and in automobiles as fuels. LPG is well-used fuel for cooking, drying and heating. Although LPG and natural gas are known as an environmental friendly but still those gaseous can instigate a serious effect in case of leakage or disclosure. Leakages may lead to explosion which can lead to many deaths. As the usage of LPG increases in domestic, the accidents occur by these LPG explosion also increases due to lack of continuous inspections and monitoring. In home residence such explosion can happens due to substandard cylinders, old valves and loose type of head gas. Other than that, configuration on weight of cylinder gas also lacking in most urban area. For example, at Kuala Lumpur, seven people were injured after two restaurant were damaged by explosions at a shopping mall. This incident occurred due to testing on restaurants' LPG piping features by two maintenance workers [1].
As the usage of LPG increases in domestic field, the accidents occur by these LPG explosion also increases due to lack of continuous inspections and monitoring. Therefore, it is necessary to have a system which continuously monitor the cylinder. The purpose of this project is to monitor and detect leakage on LPG cylinder in urban area (household applications). Commonly, there always has a sensor to detect any changes. For an example gas sensor. This sensor used to detect the amount of gas in atmosphere and alerts the consumer about this leakage by sending message through twitter or facebook and alarming. Internet of Things (IoT) system used to help owner to monitor the condition and weight of cylinder anytime and useful for replacement of new cylinders on time without any fail [2]. The Internet of Things is actually the latest technology used by people nowadays. This technology gives life to the real world things. IoT will be connected to an object like door, car, refrigerator, home with internet. This will help user to access and accumulate information of the connected devices at any time in anywhere in the world [3]. 


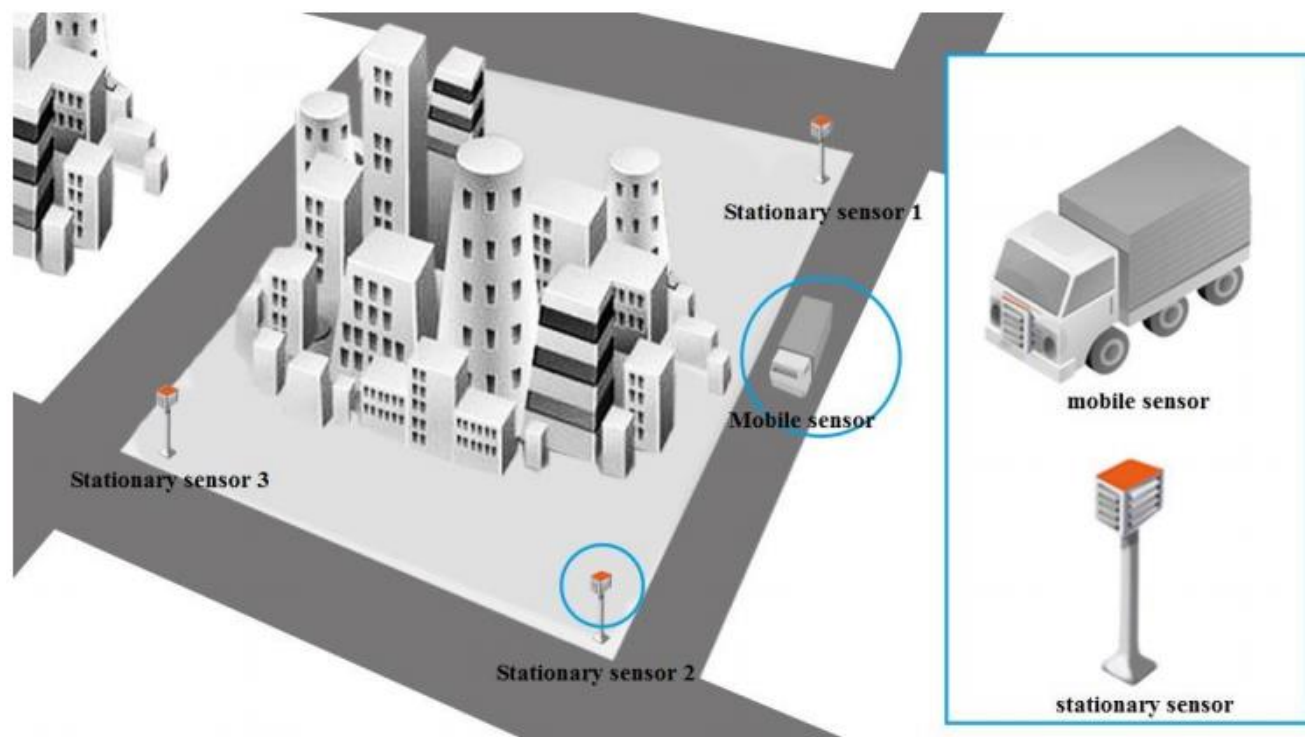

Fig.1. Online monitoring system for natural gas leakage in cities with sensor network [6]

\section{Related Works}

Traditionally, people used soapy water to detect gas leakage. Firstly, they mix few tablespoon of dish soap into a container of water. The dish soap should not contain ammonia because ammonia can cause brass to become crack and brittle. Then, they turn on the gas supply. Lastly, they wet down the spot by using a soaked sponge or spraying the soapy water on it, to see if there is bubbles form on them. If the bubble exist, the leakage spot is found. Lately, in market, there are various types of gas leakage detection system. Mostly, those detectors are used in hotels, restaurants and several homes. Different companies produces different types of detecting system according to its own features and customer needs.

Authors in [4] have developed a gas leakage monitoring system by using Arduino and Zigbee. The monitoring system is developed by using LabVIEW Graphical User Interface (GUI), Zigbee transceiver is used to monitor the gas concentration, gas detector with Liquid Crystal Display (LCD), alarm system built by using buzzer to alert the workers, and an autonomous control system is developed to trigger the exhaust fan automatically in order to ventilate the dangerous gases in the room as well as auto shut down the main power and the gas. In [5], a Global System for mobile communications (GSM) based LPG leakage detection and controlling system is developed. Once leakage of gas is detected, the valve is automatically closed to stop further gas leakage. Then the electric power supply is also shut down to prevent fire accidents. In particular, gas sensor has been used which has high sensitivity to gases like propane and butane. GSM module is used to alert users by sending SMS. On a larger scale, authors in [6] developed an online monitoring system for natural gas leakage in cities with sensor network, as depicted in Fig.1. The system consists of mobile sensor network, stationary sensor network, and cloud storage platform. The sensor network, i.e., mobile detectors and stationary detectors, are used to collect methane concentration data for the entire monitored area. The natural gas leakage monitoring cloud platform is designed to collect and analyze the methane concentration data, calculate the leakage location(s), and support the emergency response.

Compared to the related works, additional function of the monitoring system is added in this project. The proposed system is known as LPG leakage detection and monitoring system where users can be notified of the gas leakage and are able to track the weight of cylinder gas by using their smartphone at any time, and from anywhere, as long as they are connected to the internet.

Fig. 2 shows the overall concept of the proposed system in this project. In this project, two types of sensors are used. The sensors are MQ6 gas sensor which only detects LPG gas and a $40 \mathrm{~kg}$ load cell with HX711 balance module amplifier. Both sensors are connected to NODEMCU ESP8266 device. This device can connect to $\mathrm{Wi}-\mathrm{Fi}$, for IoT feature. There are two types of alarm alerts too. Physical alarm is 
developed using buzzer and non-physical alarm is developed by sending alert through user email and smartphone notification. In addition, the data of gas leakage and weight of cylinder is updated into Blynk cloud in real-time. Blynk is one type of IoT platform.

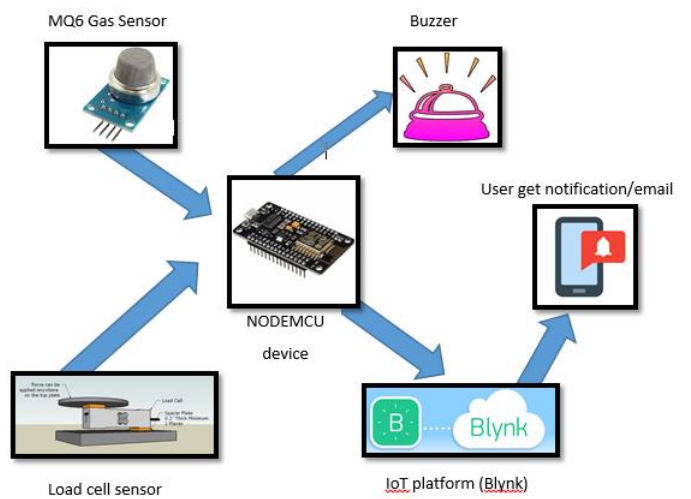

Fig. 2. Overall system setup.

\section{Experimental Setup}

Based on Fig. 3, this project is divided into four phases. In phase 1, blinking of LED light to test the NodeMCU ESP8266 and controlling the $\mathrm{ON}$ and OFF by IoT system is developed. The purpose of this phase is mostly testing on NodeMCU ESP8266 IoT system. In phase 2, the development of sensing unit using LPG gas sensor and load cell is upgraded in hardware part while in software section is the programming for both sensing unit. In phase 3, the hardware part is upgraded by developing the alert system using buzzer and the software is to build the program code for the alert system. Finally, in phase 4, the hardware development on monitoring system using IoT and alert system using email is developed and the software section involved the settings in IoT system. This phase is where user can monitor the condition and weight of cylinder through the IoT system website.

\section{A. Hardware Development}

The development of the Liquefied Petroleum Gas (LPG) leakage detection and monitoring system design is shown in Fig. 4. Most important is the brain of this system, which is the NodeMCU ESP8266 equipped with Wi-Fi connection capability. Since NodeMCU ESP8266 is embedded with a microcontroller of its own, therefore an external microcontroller is not required and the programming can be programmed by using the
Arduino IDE itself. Additionally, the detector system is designed with LPG MQ6 gas detector that detects gas leakage at surrounding area. The piezoelectric buzzer rings once the gas detector detects the gas leakage and an alert message is also sent to user via email. The system is also equipped with a load cell as a sensing unit to monitor the weight of gas cylinder and send alert message to user through his email. The HX711 balance module is connected to load cell which acts as an amplifier where it as an A/D converter. This system also employs the concept of IoT as its monitoring and alarm system. Desired data collected by NodeMCU ESP8266 are sent to the Blynk application cloud and can be accessed by the users anytime he wants.

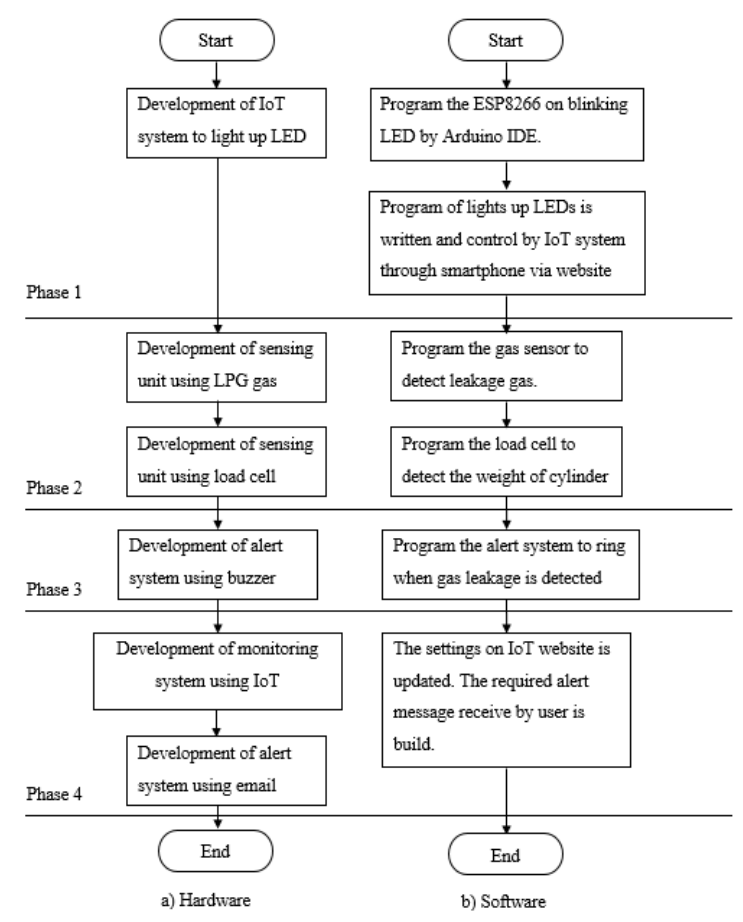

Fig. 3. Flowchart of LPG gas leakage and monitoring system for (a) hardware and (b) software.

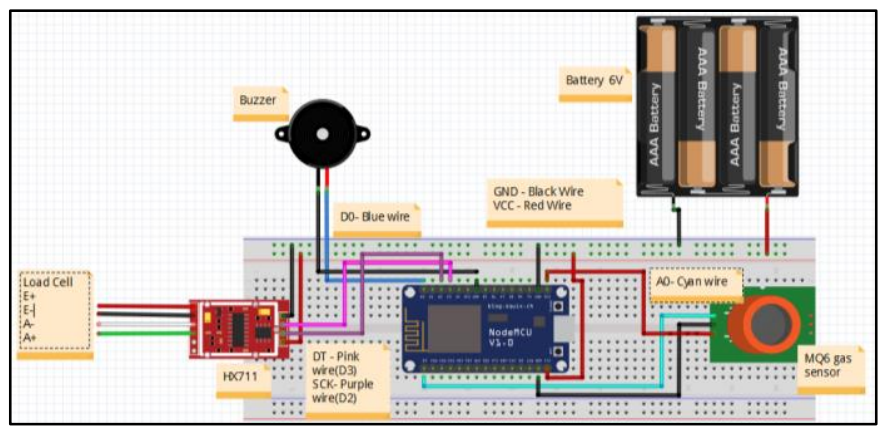

Fig. 4. Circuit diagram of LPG gas detector with monitoring system. 


\section{B. Software Development}

This section describes the programming done to the processor incorporated with sensing unit (gas sensor and load cell), alarm unit (rings the buzzer and send alert messages) and monitoring unit (update the data) through the Internet of Things (IoT) website according to phases in Fig. 3. Table 1 listed all the categories of cases considered in this project. Fig. 5 shows the programming flowchart according to the four cases in this system.

Table 1. Categories of Cases

\begin{tabular}{|l|l|l|}
\hline Case & Gas cylinder & Gas Leakage \\
\hline 1 & OK & OK \\
\hline 2 & OK & ALERT \\
\hline 3 & ALERT & OK \\
\hline 4 & ALERT & ALERT \\
\hline
\end{tabular}

Case 1 is when the kitchen area is safe, without any gas leakage in surrounding and weight of gas cylinder is still above critical stages. In this case, the alarm unit will be silent, therefore there will be no buzzer ringing or alert message send to user. However, the data is still updated to website for user monitoring purposes.

Case 2 shows the weight of cylinder is above the critical stage but with gas leakage detected. For this case, buzzer will ring and system sends alert message of 'Gas leakage detected' to user and updates the data in website.

In case 3 , the cylinder weight is below the critical stage and there is no gas leakage detected. In this case only alert message like 'New cylinder replacement' will be send to user while the buzzer or other alert message will not be triggered due to no leakage detected in surrounding. At the end, the data will be uploaded into website for monitoring purposes.

In case 4, the cylinder weight is below the critical stage and there is also gas leakage detected. In this case both alert message of 'New cylinder replacement' and 'Gas leakage detected' will be sent to user and buzzer will ring. The data will also be uploaded into website for monitoring purposes. Lastly, user will receive all the alert message through his email and he can open the Blynk application to monitor the system.

\section{Results and Discussion}

This section provides the explanation of the results and discussion. It is divides into four phases. This part explains more detail with the aid of figures the development of hardware and software. In software development, the cases is elaborated case by case according to the categories listed previously in Table 2 .

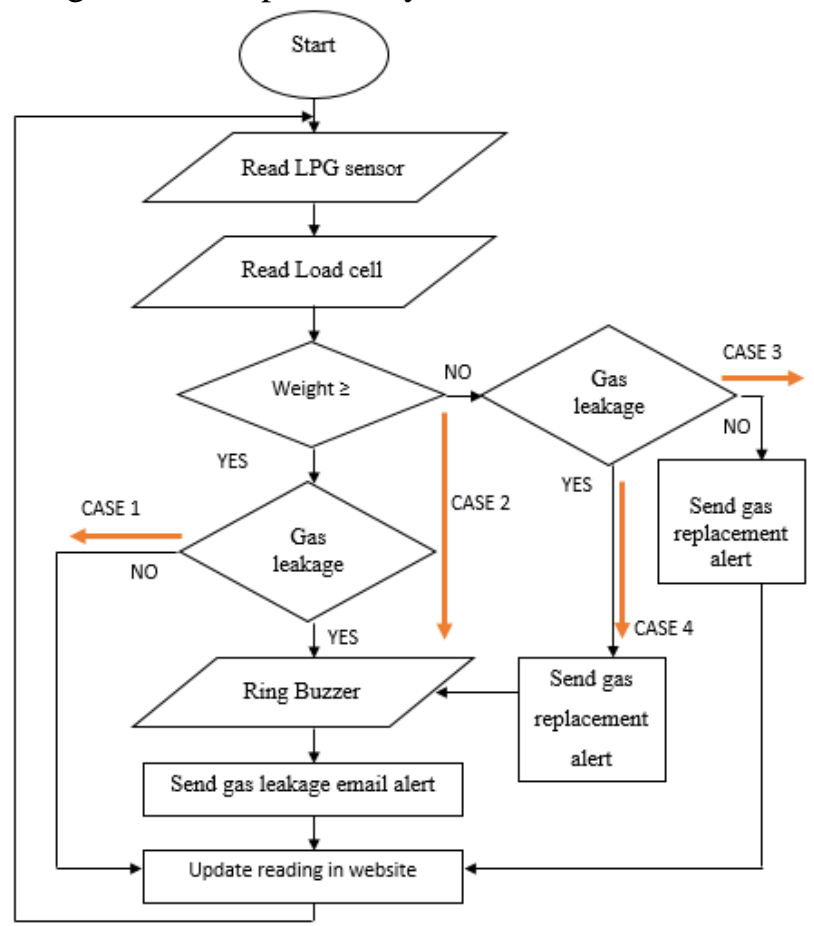

Fig.5. Flowchart for four cases.

\section{A. Development of IoT system to light up LED lights (Phase 1)}

The purpose to conduct this phase is to test the IoT platform Blynk. The testing done by turning "ON" and "OFF" a LED light as shown using Blynk application shown in both Fig. 6 and Fig. 7.

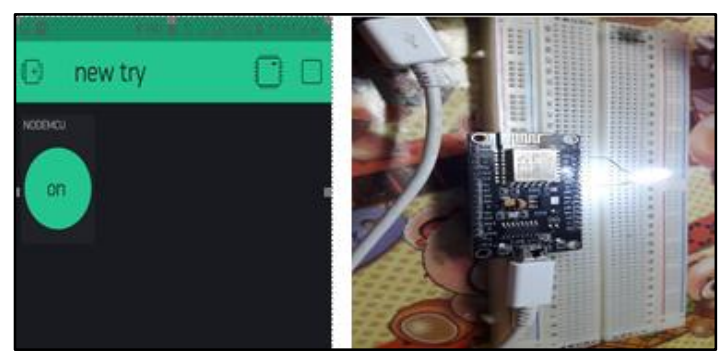

Fig.6. Press "ON" and the LED lights up. 


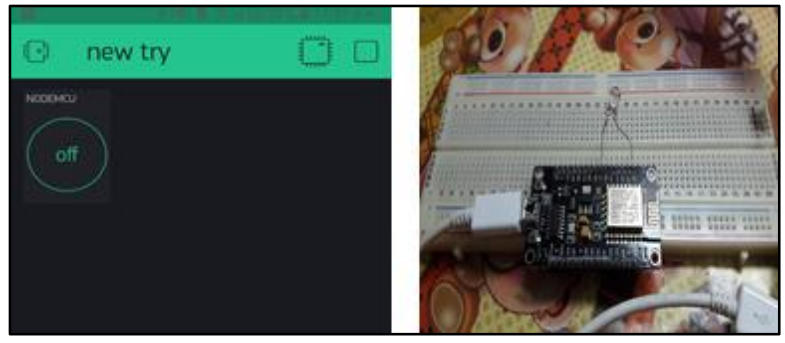

Fig. 7. Press "OFF" and the LED lights off.

\section{B. Development of sensing unit using gas sensor and load cell (Phase 2)}

The first sensing unit developed is gas sensing. This gas sensor detects the amount of LPG gas in the surrounding area. Since this project involves detection of the LPG only, therefore MQ6 gas sensor was chosen.

Fig. 8 shows the connection of gas sensor with NODEMCU ESP 8266 device. For testing the gas leakage, a lighter have been used. This is because the liquid inside the lighter is a Liquidfied Petroleum Gas. Once the gas sensor detects the LPG gas in surrounding, the serial monitor displays the amount of LPG gas leaked.

Table 2 shows the collection of data taken in two different area. This both place done in the kitchen area around $600 \mathrm{~cm} \mathrm{X} 390 \mathrm{~cm}$ and cabinet area around $100 \mathrm{~cm} \times 250 \mathrm{~cm}$. The testing at open area carried near the stove. The gas sensor have been placed according to the stated distance. Then, a timer about 60 s have been set. Next, the stove was turn on to leak the LPG gas to the surrounding. The reading that display in the serial monitor have been recorded. The experiment have been repeated for three times so that an average data can be calculated. Based on the data observed, the device can be placed in $30 \mathrm{~cm}$ distance and the threshold value is set to be 100 as the maximum amount of leakage per minute.
Next, load cell is used to measure the weight of gas cylinder. A $40 \mathrm{~kg}$ load cell is chosen because the total weight of a C14 LPG gas cylinder with gas is $30 \mathrm{~kg}$ to $32 \mathrm{~kg}$ while empty tank is around $15.9 \mathrm{~kg}$ to $16.4 \mathrm{~kg}$. The weight of empty tanks are different according to its height. However, the amount of gas fill up in each tank is standard as $14 \mathrm{~kg}$.

Firstly, a calibration step is carried out. This step is important to calibrate the load cell to read the correct reading. The LPG gas cylinder is weighed using an electronic weighing scale as reference, shown in Fig. 9. This is to make sure that the load cell reading after calibration is matched with the reference weighing scale reading. After few trials, calibration factor value is set to be 132026 .

Fig. 10 shows the setup of load cell with the NODEMCU ESP 8266 and an empty gas cylinder is placed on top the load cell. The load cell is connected to HX711 amplifier, then this amplifier connected to NODEMCU ESP8266 device. The value read by a load cell is in analog form. Fig. 11 shows the reading of load cell, which conforms to the reference in Fig. 9. Therefore, the calibration factor have been fixed for entire project.

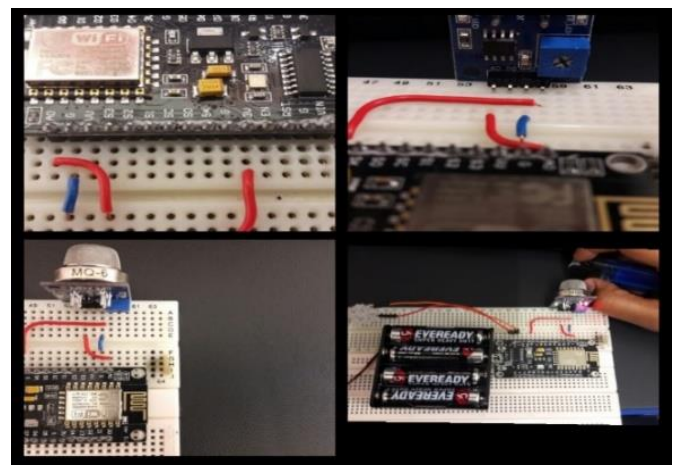

Fig. 8. Connection and testing of MQ6 gas sensor.

Table 2.

\begin{tabular}{|c|c|c|c|c|c|c|c|c|}
\cline { 2 - 9 } \multicolumn{1}{c|}{} & \multicolumn{4}{c|}{ OPEN AREA (600CM*390CM) } & \multicolumn{3}{c|}{ CLOSED AREA (100CM*250CM) } \\
\cline { 2 - 9 } \multicolumn{1}{c|}{} & $1 \mathrm{ST}$ & $2 \mathrm{ND}$ & $3 \mathrm{RD}$ & AVERAGE & 1ST & 2ND & 3RD & AVERAGE \\
\hline $10 \mathrm{CM}$ & 110 & 112 & 109 & 110.33 & 121 & 128 & 138 & 129 \\
\hline $20 \mathrm{CM}$ & 98 & 99 & 100 & 99 & 116 & 120 & 113 & 116.33 \\
\hline $30 \mathrm{CM}$ & 85 & 86 & 90 & 87 & 92 & 82 & 95 & 89.67 \\
\hline $40 \mathrm{CM}$ & 84 & 85 & 85 & 84.67 & 65 & 71 & 69 & 68.33 \\
\hline $50 \mathrm{CM}$ & 80 & 75 & 79 & 78 & 54 & 55 & 52 & 53.67 \\
\hline
\end{tabular}




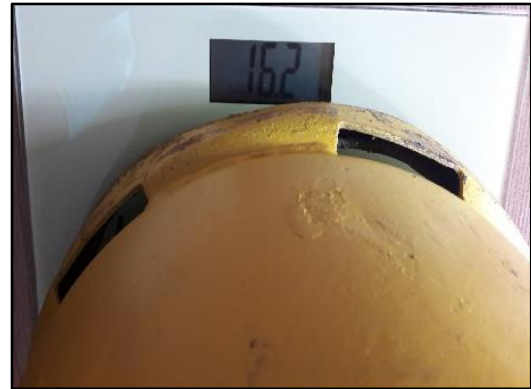

Fig. 9. LPG gas cylinder weighted using weighing scale.

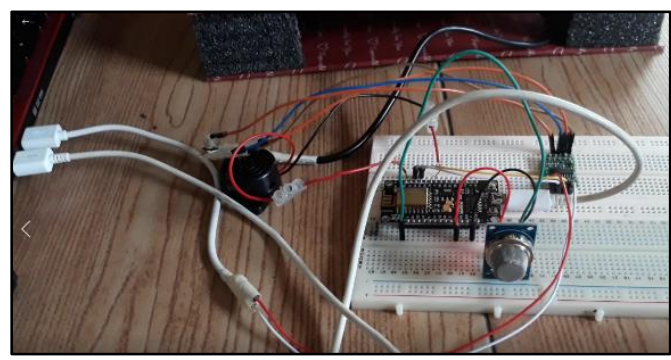

Fig. 10. Setting of load cell with device for calibration and testing.

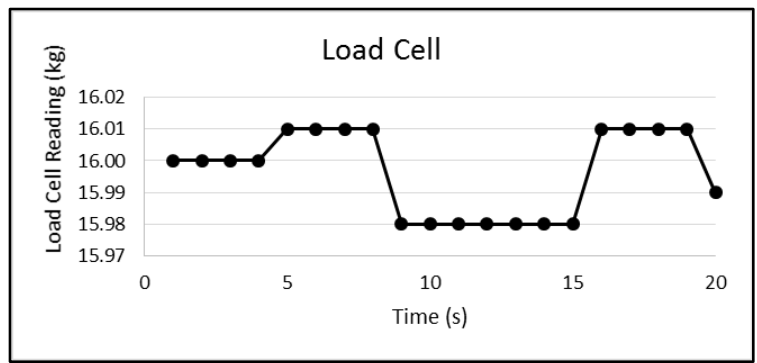

Fig. 11. Load cell reading of an empty tank reading after calibration.

\section{Development of Alert System using Buzzer}

In this phase, a buzzer is added into gas sensor circuit. Since this project consists of physical alarm, therefore buzzer is the audible alarm for user. In addition, this phase is known as alert system phase. The connection of buzzer with a NodeMCU ESP8266 is shown in Fig. 12. Buzzer rings when the gas sensor detects high LPG gas reading from the surrounding area. The detection based on the threshold value that have been stated in the coding about 100 . Once the gas sensor value is equal to or more than 100 , the buzzer is turned On.

\section{Development of Monitoring System using Internet of Things (IoT)}

The advantage of this project as compared to others is the online monitoring system based on latest technology of Internet of Things (IoT). This is the last phase for this project. In this phase, there are two sections. The first is the development of monitoring system using Internet of Things via Blynk app. The second part is the upgraded of non-physical alert system such as notification and email.

Fig. 13 shows an example of readings shown in Blynk during testing of this device. The LPG GAS gauge displays the amount of gas leaking in surrounding while LOAD CELL gauge will displays the weight of gas cylinder.

Another important part of this project is the non-physical alert system. This system consists of both notification and email alert alarm. In Blynk, the alert alarm system is built by using an Eventor. In the "new try" screen, an email icon and notification icon is added. The nonphysical alert system is tested according to the cases.

Case 1: In this case, the alert system remained silent. This is because there is no gas leakage and also the cylinder weight is more than 20kg. Since both sensor shows the condition is in OK state, therefore user would not receive any alert alarm notification.

Case 2: In case 2, the alert condition triggered is from the gas sensor which means there is LPG gas leaking in the surrounding. In this case both alarm alerts is turned on. The buzzer will start to ring and at the same time Blynk will send notification and email to user as "AN ALERT!!! Take an Action!!! LPG gas is leaking!!!” as shown in Fig. 14.

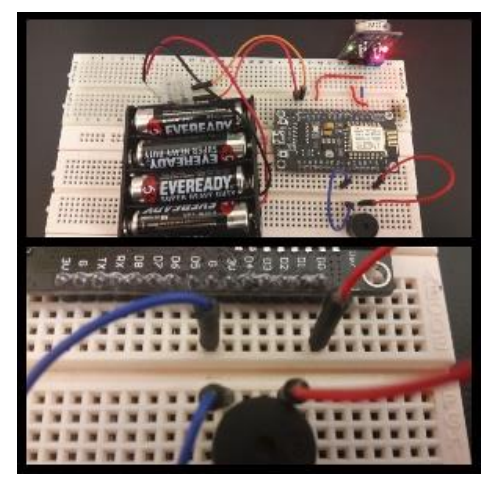

Fig. 12. Setting for buzzer.

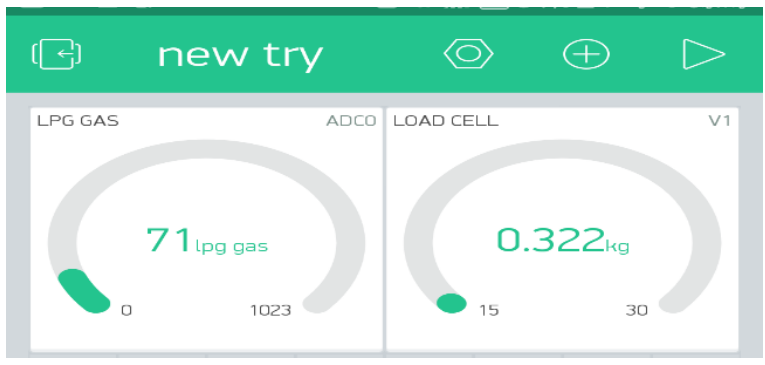

Fig. 13. Two types of gauges such as LPG GAS and LOAD CELL in Blynk. 


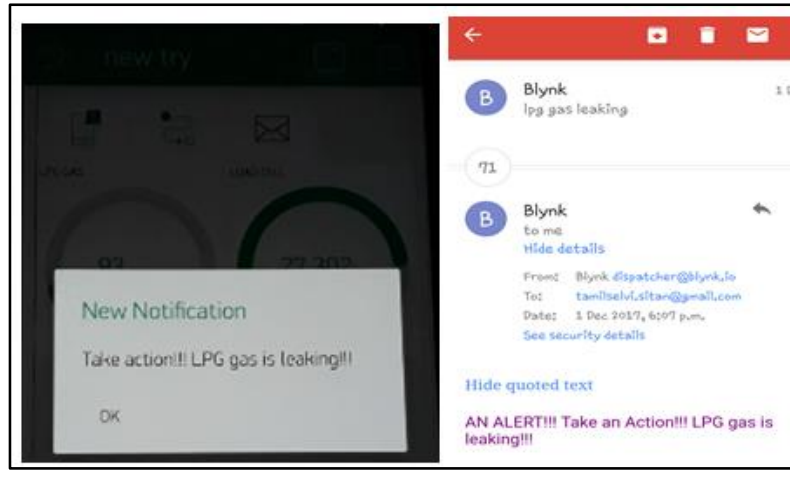

Fig. 14. The alert message send by Blynk to user.

Case 3: In this case, load cell sensor triggered the alarm. This means that the gas cylinder is lighter or equal to $20 \mathrm{~kg}$, which indicated that only quarter tank is left. Therefore, Blynk sent an alert message to user as "AN ALERT!!! Replacement of cylinder gas!!!" as shown in Fig. 15. In this case, buzzer will remain silent because there is no gas leaking. Buzzer alarm is only for gas leaking alert.

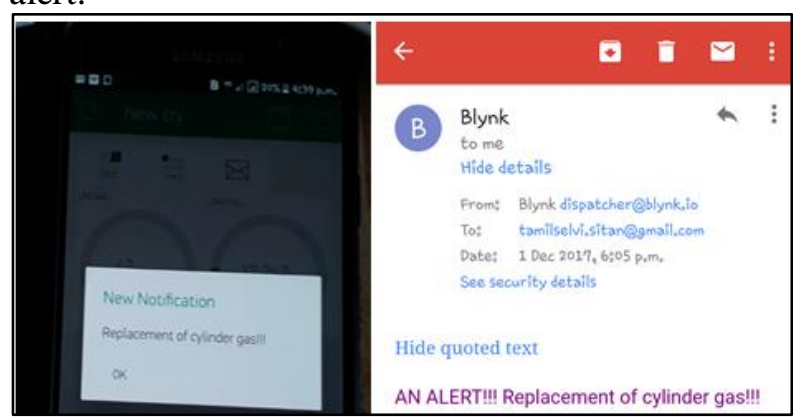

Fig. 15. The alert message send by Blynk to user

Case 4: Case 4 is the case where both gas and load cell sensors triggered the alert condition in Blynk and buzzer. In this case, both physical and non-physical alarm will turn on. Once the gas sensor detects the spike in LPG reading from its surrounding, buzzer is automatically turned $\mathrm{ON}$. At the same time, Blynk sent alert message to user as "AN ALERT!!! Take an Action!!! LPG gas is leaking!!!" and "AN ALERT!!! Replacement of cylinder gas!!!" as shown in Fig. 16.

A simple analysis has been conducted to test the coverage of gas leakage detection. The sensing unit is found to be capable of detecting gas leakage at about $30 \mathrm{~cm}$ distance in both open and closed area. Besides that, the alert system is found to be very prompt, where users receive the email notification only seconds after the detection. Hence, the system can be classified as real-time.

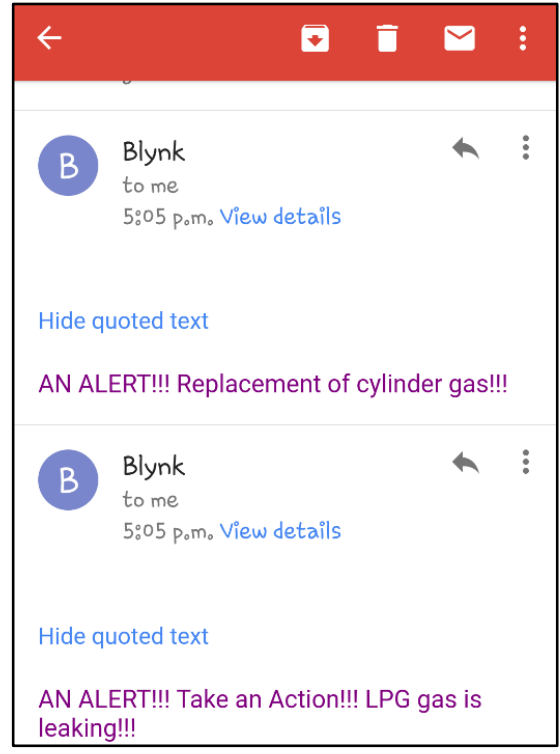

Fig. 16. The alert message send by Blynk to user.

\section{Conclusion}

In a nutshell, a Liquefied Petroleum Gas (LPG) Detector and Monitoring system has been successfully developed. The sensing unit has successfully monitored weight of gas cylinder and detected the gas leakage in surrounding. In additional, the physical and non-physical alert alarm is working successfully according to the given command. Besides that, the MQ6 gas sensor able to detect the gas leaking in open and closed area about $30 \mathrm{~cm}$ in distance. Furthermore, the online monitoring system via IoT platform Blynk is also successfully developed. Hence, user can monitor the gas leakage and weight of gas cylinder in Blynk through their smartphone whenever and wherever they wants.

\section{Acknowledgements}

This work was supported by Ministry of Higher Education (MOHE), Faculty of Engineering Technology, Research Management Centre, Universiti Tun Hussein Onn Malaysia (UTHM) under Grant (Vote No: FRGS 1619).

\section{References}

[1] S. B. Mun, (2016) Mid Valley gas-leak explosion injures 8, The Sun Daily, 05-Apr2016. 
[2] H. Ruqsar, R. Chandana, R. Nandini, and T. Surekha, (2014) Internet of Things ( Iot ) Based Real Time Gas Leakage Monitoring and Controlling, Int. J. Electron. Commun. Eng. Technol., vol. 5, no. 8, pp. 208-214.

[3] I. Lee and K. Lee, (2015) The Internet of Things (IoT): Applications, investments, and challenges for enterprises, Bus. Horiz., vol. 58, no. 4, pp. 431-440.

[4] H. H. Yan and Y. Rahayu, (2014) Design and development of gas leakage monitoring system using arduino and zigbee, Proceeding Electr. Eng. Comput. Sci. Informatics, vol. 1, no. 1, pp. 207-212.

[5] M. Amsaveni, A. Anurupa, R. S. A. Preetha, C. Malarvizhi, and M. Gunasekaran, (2015) Gsm based LPG leakage detection and controlling system, Int. J. Eng. Sci. ISSN, pp. 1813-2319.

[6] Y. Liu, P. Yang, G. Wang, C. Liu, and J. Qian, (2015) Online monitoring system for natural gas leakage in cities with sensor network, in Proceedings of the 1st ACM SIGSPATIAL International Workshop on the Use of GIS in Emergency Management, pp. 14. 